\title{
Synthesis of PbTe nanocubes, worm-like structures and nanoparticles by simple thermal evaporation method
}

\author{
L KUNGUMADEVI and R SATHYAMOORTHY* \\ PG and Research Department of Physics, Kongunadu Arts and Science College, Coimbatore 641 029, India
}

MS received 6 March 2012; revised 29 June 2012

\begin{abstract}
Nanocrystalline PbTe thin films are prepared by thermal evaporation on glass substrates. The investigations of X-ray diffractograms have shown that the structure of film is found to possess stable face centred cubic $(f c c)$ phase in which the grains predominantly grow in the direction of (200) plane. The grain size of the films is within the range of $27-43 \mathbf{~ n m}$. Morphologies like assembly of nanoparticles, worm-like structures and nanocubes were prepared by tuning the film thickness. Electrical resistivity is measured using four-probe technique and its thickness dependence has been analysed on the basis of 'effective mean free path model'. A change in conductivity from $n$-type to $p$-type is observed due to the increase of migration of tellurium vacancies in the films with temperature. Bandgap energy of the PbTe nanocrystalline thin films suffered a large blue shift of about $1.299 \mathrm{eV}$ due to quantum confinement of charge carriers. The nanocrystalline PbTe thin films of different morphologies such as nanoparticles, wormlike and nanocubes have the optical bandgap energies of 1.61, 1.23 and 1.01 eV, respectively. Photoconductivity measurement shows that the prepared nanocrystalline PbTe thin films of different morphology exhibits good response. This structure induced change in optical properties may have potential applications in optoelectronics devices.
\end{abstract}

Keywords. Semiconductor (PbTe); nanostructures; X-ray diffraction; morphology; resistivity; quantum confinement.

\section{Introduction}

Semiconductor nanocrystalline thin films have attracted much attention in recent years in both fundamental research and technological applications, because of their tunable sizedependent electronic and optical properties. For the past few decades, the synthesis of low-dimensional semiconductor materials with controlled morphologies like quantum dot, nanorod, nanowire, etc. finds applications in optoelectronics, biotechnology, catalysis, etc (Zhang et al 2003, 2005). Lead chalcogenide materials exhibit properties which are unusual and possibly unique, relative to other semiconductors and these unusual characteristics have obviously added to the stimulated study. Particularly, unusual feature of this group of materials is the relative stability of the lattice over a wide range of non-stoichiometry. The temperature coefficient $\left(d E_{\mathrm{g}} / d T\right)$ for these compounds is positive while it is negative for all other semiconductors. The semiconductors, $\mathrm{PbS}, \mathrm{PbSe}$ and $\mathrm{PbTe}$, show a small direct gap at $\mathrm{L}$ point of Brillouin zone. In contrast to most semiconductors, this gap decreases with hydrostatic pressure and increases with temperature. Further, the static dielectric constants of lead chalcogenides are unusually large when compared with values for other semiconductors (Kumar et al 2005). As one of the important IV-VI semiconductor materials, the rock-salt

\footnotetext{
*Author for correspondence (rsathya1959@gmail.com,
} rsathya59@gmail.com) (face centred cubic) structured lead telluride $(\mathrm{PbTe}$ ) nanostructure has been the object of particular attention, because of its narrow bandgap of $0.32 \mathrm{eV}$ (in the bulk at room temperature), tunable absorption edge, large molar extinction coefficient, multiple exciton generation and strong quantum confinement effect owing to its large Bohr radius (about $r_{\mathrm{B}}=150 \mathrm{~nm}$ ) (Ma et al 2009), and hence, potential for applications in novel photoelectric devices (Lin et al 2012). Only a very few reports are available for synthesizing nanostructured $\mathrm{PbTe}$ thin films by vacuum evaporation (Wang et al 2008). In this paper, we report structural, electrical, optical and photoconduction properties of thermally evaporated nanocrystalline $\mathrm{PbTe}$ thin films.

\section{Experimental}

In order to produce high quality $\mathrm{PbTe}$ thin films by thermal evaporation, the glass substrates were methodically cleaned prior to film deposition starting with $\mathrm{NaOH}$ solution followed by ultrasonic cleaning in distilled water and isopropyl alcohol, sequentially. A molybdenum boat was charged with PbTe powder (99.998\% pure, Aldrich Chemicals). The vacuum chamber was pumped down to $2 \times 10^{-5}$ torr. The boat was gradually heated until the material started to evaporate. The vapour molecules leaving the source are deposited onto the substrate surface. The rate of deposition was $1 \AA / \mathrm{s}$ and thickness of the films was monitored by a conventional 
quartz crystal monitor. The substrates were held at room temperature during the deposition process.

$\mathrm{X}$-ray diffraction measurements were performed on a Shimadzu XRD 6000 X-ray diffractometer using $\mathrm{CuK} \alpha$ $(\lambda=1.54056 \AA)$ radiation. A JEOL JSM 6330f scanning electron microscope (SEM) was employed to analyse surface morphology of the PbTe films. Electrical resistivity measurements in the temperature range of 300-463 K were carried out using four-probe method. Optical investigations were performed using spectrophotometer (Jasco Corp., $\mathrm{V}-570$ ) which allowed measurement in the spectral range of 200-2500 nm with $1 \mathrm{~nm}$ resolution. Photoconduction measurements were performed using Keithley 2612-SMU at room temperature under a rotary vacuum.

\section{Results and discussion}

\subsection{X-ray diffraction}

X-ray diffractograms of nanocrystalline PbTe films of different thickness are shown in figure 1. The existence of sharp peaks in diffractograms suggests the polycrystalline nature of films. The observed $d$-spacing and $h k l$ planes are in good agreement with JCPDS (78-1905) X-ray file data of cubic PbTe, confirm the rock salt $(\mathrm{NaCl})$ structure of PbTe film. From XRD, it can be inferred that PbTe films are grown with a preferential orientation along (200) plane. The absence of any diffraction peaks related to elemental $\mathrm{Te}$ or $\mathrm{Pb}$ is another sign of quality of the films. A broad hump observed in the diffractogram of lower thickness film can be attributed to the glass background. For the film thickness of $220 \mathrm{~nm}$, this background is almost eliminated and the crystallites are grown preferentially along (200) plane. However, for a thickness of $500 \mathrm{~nm}$, we observed a significant decrease in the value of $(200) /(220)$ intensity ratio. This suggests that film grown at higher thickness is completely recrystallized (Zhao and Qi 2006).

The grain size $(D)$, dislocation density $(\delta)$, microstrain $(\varepsilon)$ and lattice constant of the cubic PbTe nanocrystals were calculated (Kungumadevi et al 2008) and are given in table 1. The grain size of the films is within the range of $27-$ $43 \mathrm{~nm}$ which indicates that the films are nanocrystalline and increases with increase in film thickness. The variation of lattice parameter as a function of thickness is presented in table 1 . It can be seen that the lattice parameter ' $a$ ' increases with the increase in film thickness and decreases on further increase in film thickness, from which the influence of thickness on the lattice parameter is clearly observed. Interestingly, for film thickness of $500 \mathrm{~nm}$, a value of $6.456 \AA$ was observed, which is very close to JCPDS value for bulk PbTe, i.e. $6.454 \AA$. This suggests that at higher thickness, the film is completely recrystallized yielding unstrained lattice and it is also evident from the decrease of (200)/(220) intensity ratio as discussed earlier. As thickness increases, there is an increase in grain size and decrease in strain as well as dislocation density. These results indicate a quality of the degree of crystallinity of the films, which increases with film thickness. This improvement in crystallinity is generally due to an increased ability of adatoms to move towards stable sites in the lattice. In addition, the trend of an increase in grain size with increasing film thickness may be interpreted in terms of a columnar grain growth in the structure.

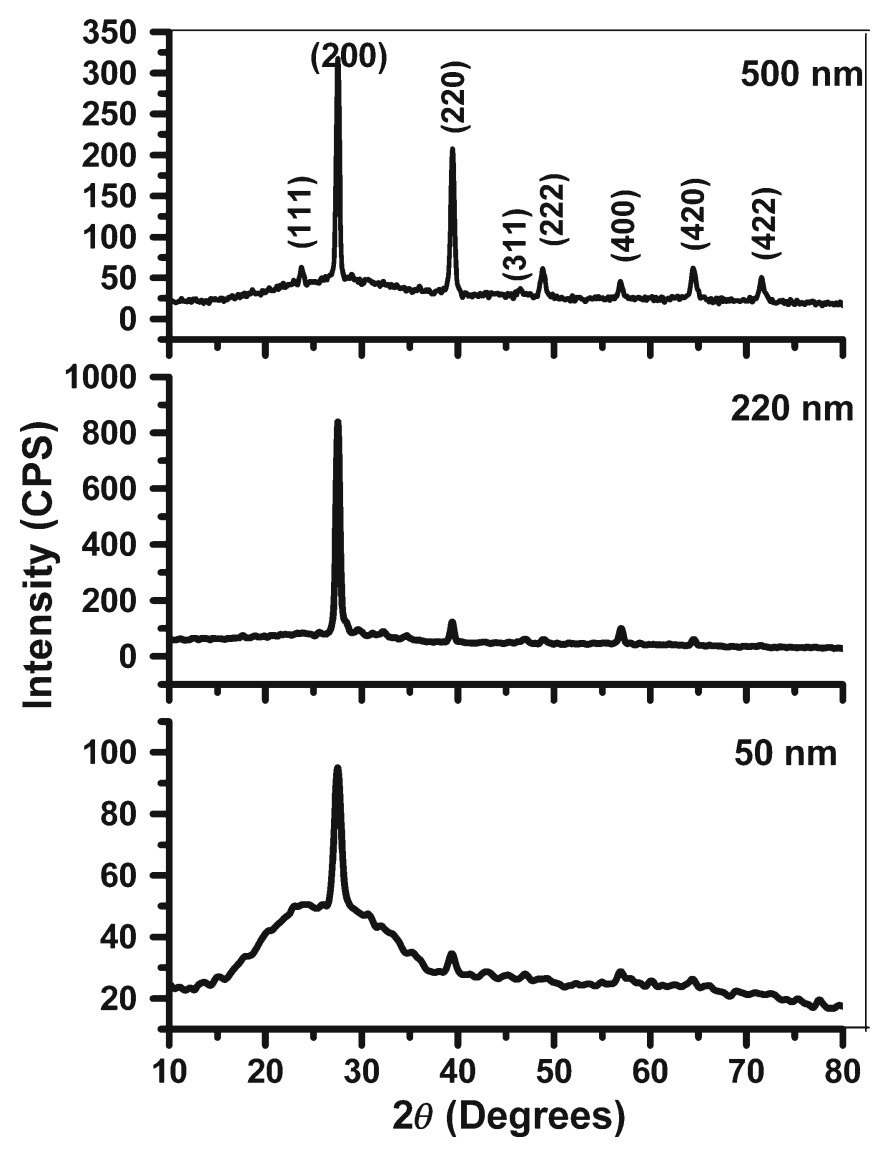

Figure 1. XRD patterns of nanocrystalline PbTe thin films of different thicknesses.

Table 1. Structural parameters of nanocrystalline PbTe thin films.

\begin{tabular}{rcccc}
\hline $\begin{array}{l}\text { Film thickness } \\
(\mathrm{nm})\end{array}$ & $\begin{array}{c}\text { Lattice constant } \\
(\AA)\end{array}$ & $\begin{array}{c}\text { Grain size } \\
(\mathrm{nm})\end{array}$ & $\begin{array}{c}\text { Strain }(\varepsilon) \\
\left(10^{-4}\right)\end{array}$ & $\begin{array}{c}\text { Dislocation density }(\delta) \\
\left(10^{14} \text { lines } / \mathrm{m}^{2}\right)\end{array}$ \\
\hline 50 & 6.458 & 27.86 & 14.024 & $15 \cdot 811$ \\
220 & 6.461 & $31 \cdot 26$ & $13 \cdot 860$ & $14 \cdot 160$ \\
500 & 6.456 & $43 \cdot 81$ & $10 \cdot 110$ & $9 \cdot 543$ \\
\hline
\end{tabular}




\subsection{Surface morphology}

Figure 2(a) shows SEM image of nanocrystalline PbTe thin film of thickness $50 \mathrm{~nm}$. It is observed that the film is smooth, homogeneous, fine grained and well covered to the substrate. SEM image of the film thickness of $220 \mathrm{~nm}$ (figure 2(b)) shows that a large number of particles are uniformly distributed throughout the surface. The nanocrystallites exhibit worm-like and flake shapes. Length and width of the worm like shape is around 100 and $20 \mathrm{~nm}$, respectively. Similar result is observed by Hema Chandra et al (2007) for SnSe thin films, prepared at different substrate temperatures. For the film thickness of $500 \mathrm{~nm}$ (figure 2(c)), the grain growth is prominent and well-defined cubic nanocrystallites
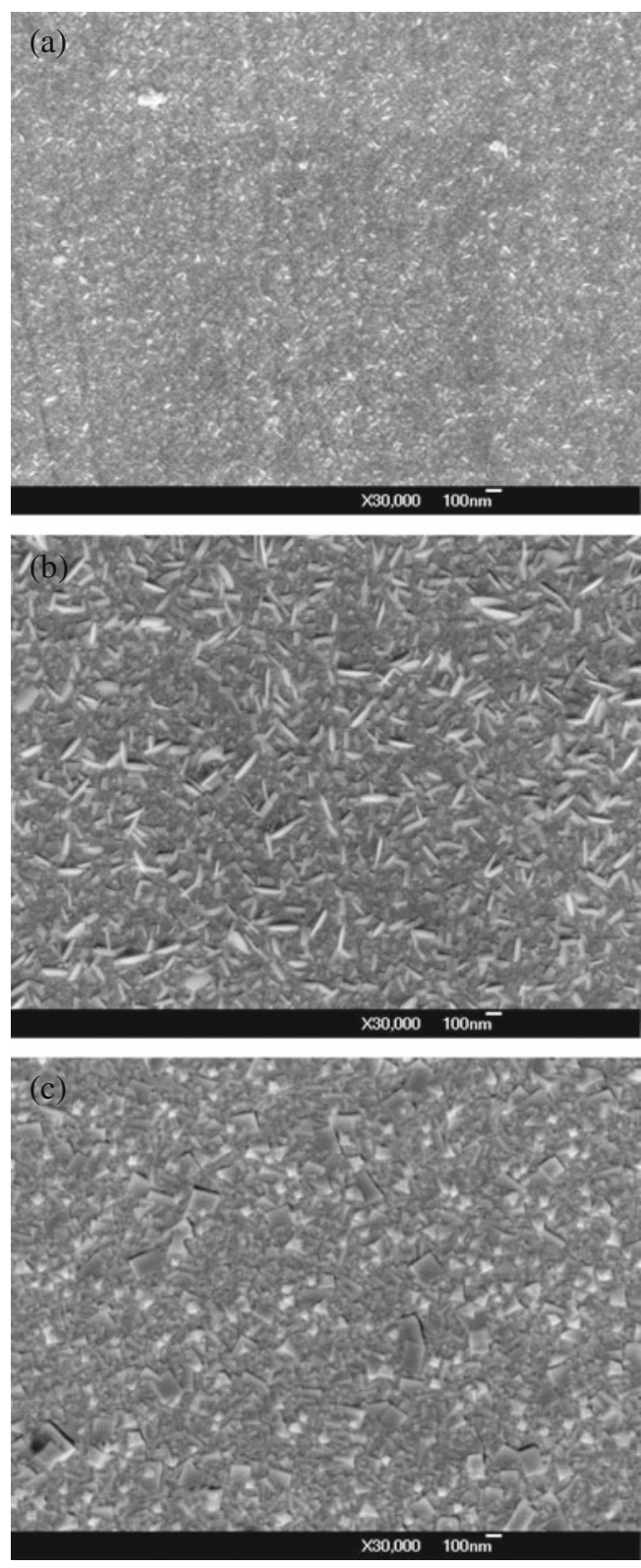

Figure 2. SEM image of nanocrystalline PbTe thin film of thickness (a) $50 \mathrm{~nm}$, (b) $220 \mathrm{~nm}$ and (c) $500 \mathrm{~nm}$. are observed. Simply by tuning the thickness, thin films with assembly of nanoparticles, worm-like structures and nanocubes of smooth surface morphology could be prepared in well-controlled manner.

SEM images indicate that the density of dislocations is not uniform which decreases as the thickness increases. This may be due to the tendency of accumulation of material, leading to group together for the formation of walls (subgrain boundaries) surrounding small volumes. As a result of this behaviour, there may be reduction of the dislocation density and improvement of the grain size. It is noted from SEM images that due to the increase in film thickness there is an obvious improvement in the grain size as observed earlier from XRD analysis (table 1).

\subsection{Electrical resistivity}

Variation in the electrical resistivity of a semiconductor depends on the changes in carrier concentration and mean free path of the charge carriers. The charge carriers are reflected/scattered by the surface of the material when they reach it. When the specimen becomes thin enough, i.e. thickness is comparable with the mean free path of the charge carriers, the collisions of charge carriers with the surface are a significant fraction of the total number of collisions. So, we can expect that the resistivity will be thickness dependent (at a constant temperature), when the thickness of the specimen is comparable with the mean free path of the charge carriers. In order to study the thickness dependence of resistivity of the films, the resistivity is plotted against inverse thickness of the film at different temperatures (figure 3). It is seen from figure 3 that the resistivity varies with thickness as expected and also it varies linearly with reciprocal of thickness. As XRD observations show the growth in grains with film thickness, as noted earlier, leads to reductions in grain

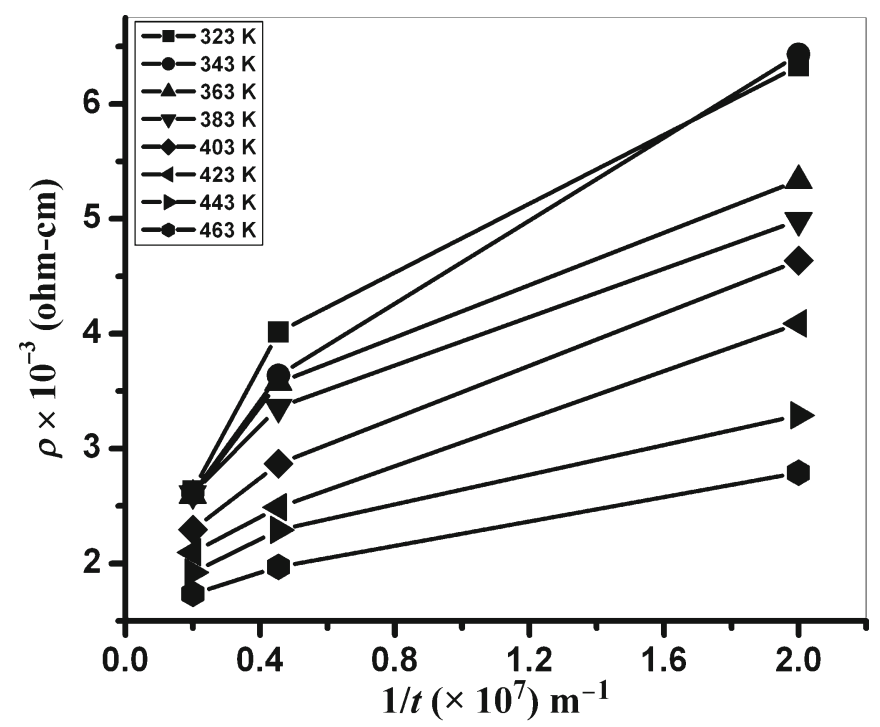

Figure 3. Variation of resistivity $(\rho)$ with reciprocal of thickness $(1 / t)$. 
boundary scattering of charge carriers, which increase the carrier mobility in the deposited films (Lee et al 2004) and eventually reduce the film resistivity. To explain the thickness dependence of resistivity, various size effect theories have been developed.

Thomson is the first to propose a size-effect theory to explain the observed high resistivity in thin specimens as compared with that of the bulk. Size-effect theory for the free electron model is worked out by Fuchs for a spherical Fermi surface and almost all subsequent work in this area has been based on his approach. In particular, Sondheimer (1950) extended the theory to include other mean free path effects (Damodara Das and Bahulayan 1996). However, Sondheimer (1950) does not take into account scattering from the grain boundary surfaces. Later, Mayadas and Shatzkes (1970) extended size-effect theory to polycrystalline films taking account also of grain boundary scattering. Tellier (1978) simplified the expression obtained from the model of Mayadas and Shatzkes (1970) and suggested a model viz. 'the effective mean free path model' by defining an effective mean free path for carriers in an infinitely thick film. According to this model, an expression for the film resistivity as a function of thickness can be obtained as (Tellier 1978):

$$
\rho=\rho_{0}\left[1+\frac{3}{8 k_{\mathrm{g}}}(1-P)\right],
$$

where $k_{\mathrm{g}}=t / \lambda_{0}$ is the reduced thickness of the polycrystalline film, $\rho_{0}$ and $\lambda_{0}$ are the resistivity and effective mean free path of the infinitely thick polycrystalline film, $t$ the thickness and $P$ the specularity parameter giving the fraction of electrons incident on the surface that are specularly scattered. Hence, if we plot $\rho$ against $1 / t$, the intercept will give the value of $\rho_{0}$, the grain boundary resistivity and the slope will give $\frac{3}{8} \rho_{0} \lambda_{0}(1-p)$. Once if we know the value of $\lambda_{0}$, we can calculate the specularity parameter, $P$ and vice versa. By assuming that the specularity parameter $P=0$ (complete diffuse scattering from surfaces), $\lambda_{0}$ is calculated from the slopes. The calculated values of $\rho_{0}$ and $\lambda_{0}$ at different temperatures are given in table 2 . It is seen that the mean free path has a dependence on temperature. It is quite interesting that the mean free path ' $\lambda_{0}$ ' decreases with increase in temperature up to $363 \mathrm{~K}$ and increases at $383 \mathrm{~K}$ and with further increase in temperature, it decreases. Increase of $\lambda_{0}$ may be attributed to transition of conduction from $n$-type to $p$-type.

The variation of resistivity as a function of temperature is shown in figure 4 . It indicates that all the films have negative temperature coefficient of resistance which suggested their semiconducting nature. This is in good agreement with earlier reports (Brown et al 1970; Seetharama Bhat and Damodara Das 1985; Mandale 1991). The activation energy for electric conduction is calculated from the slope of the lines and is presented in table 3. It can be seen that the activation energy decreases with increase in film thickness. Some structural changes, caused by defects and impurities,

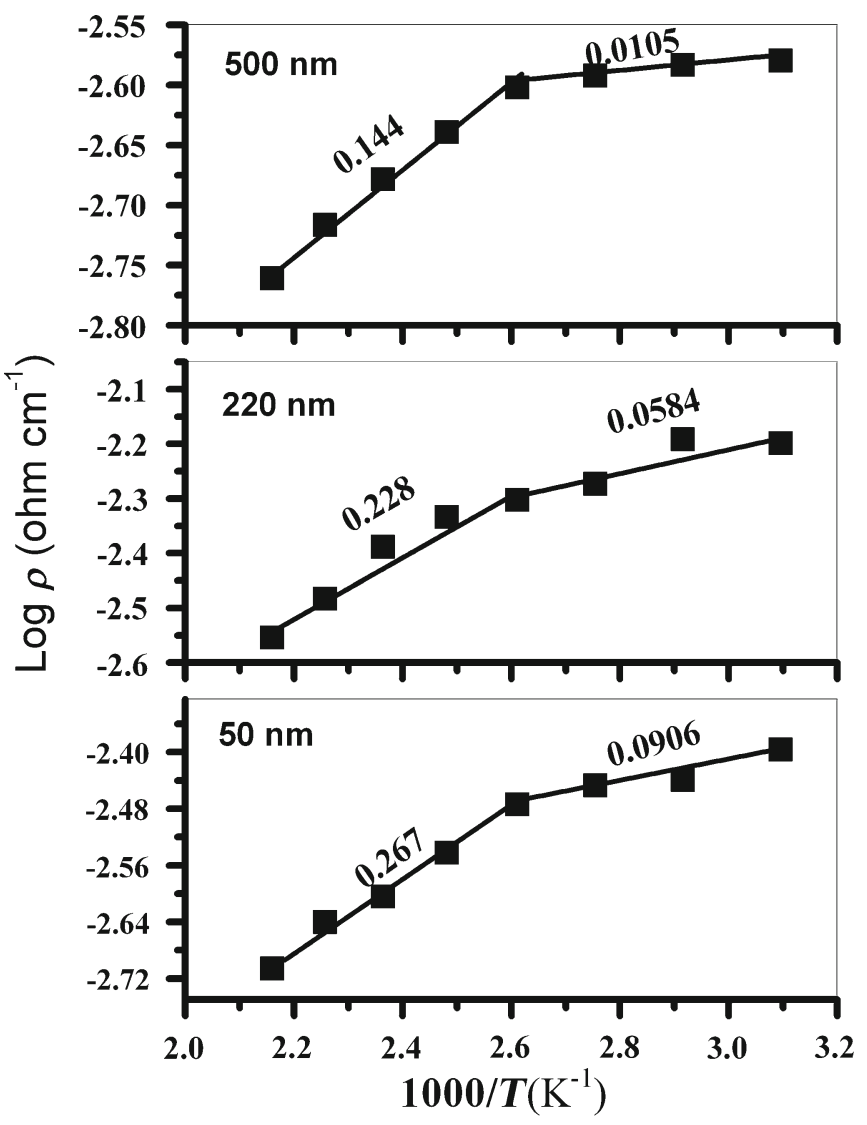

Figure 4. Plots of $\log \rho$ vs $1000 / T$ for nanocrystalline $\mathrm{PbTe}$ thin films.

Table 2. Variation of mean free path and resistivity with temperature.

\begin{tabular}{lcc}
\hline $\begin{array}{l}\text { Temperature } \\
(\mathrm{K})\end{array}$ & $\begin{array}{c}\rho_{0} \times 10^{-3} \\
\left(\mathrm{ohm}-\mathrm{cm}^{-1}\right)\end{array}$ & $\begin{array}{c}\lambda_{0} \\
(\AA)\end{array}$ \\
\hline 323 & 2.75 & 2301 \\
343 & 2.55 & 1860 \\
363 & 2.45 & 1590 \\
383 & 2.15 & 1822 \\
403 & $2 \cdot 05$ & 1672 \\
423 & 1.90 & 1603 \\
443 & 1.70 & 1176 \\
463 & 1.55 & 1075 \\
\hline
\end{tabular}

Table 3. Variation of activation energies for PbTe films of different thicknesses.

\begin{tabular}{lcc}
\hline & \multicolumn{2}{c}{ Activation energy (eV) } \\
\cline { 2 - 3 } Film thickness (nm) & Below 383 K & Above 383 K \\
\hline 50 & 0.090 & 0.267 \\
220 & 0.058 & 0.228 \\
500 & 0.010 & 0.144 \\
\hline
\end{tabular}


in the films can lead to a decrease in conductivity and the observed high temperature conductivity can be attributed to the thermal excitation of charge carriers from grain boundaries to neutral regions of the grains. The decrease in activation energy can be attributed to a decrease in defect levels. The grain boundary scattering contribution is expected to decrease significantly as the film thickness increases. Similar observations have been reported by Abd El-Ati (1997) for PbTe thin films. The observed lesser conductivity in thinner films can be explained by the lower degree of crystallinity and smaller grain size as observed by XRD analysis.

It has been found that the activation energy is thickness dependent and decreases with increasing thickness. This can be explained by the polycrystalline nature of the films, which is detailed with a model established by Seto (1975). A polycrystalline film material contains a large number of microcrystallites separated by grain boundaries that play an important role between crystallites in determining the conductivity of a polycrystalline film. The incomplete atomic bonding at a grain boundary can act as trap centres. These centres trap the charge carrier there, to build a local space charge. This charge then impedes the transition of charge carriers from one crystalline to another. Thus, the values of activation energy will vary with applied voltage, which is about different trapping levels situated between the valence and conduction bands. As the thickness decreases, crystallite size decreases and this leads to an increment in the trapping states at grain boundary. Trapping states are capable of trapping free carriers and as a consequence, more free carriers become immobilized as trapping states increase. In other words, larger crystallite size results in a lower density of grain boundaries, which behave as traps for free carriers and for carrier transport in the film. Hence, an increase in grain size can cause a decrease in grain boundary scattering, which leads to an increase in conductivity. This is because the crystallinity of the films is improved and the grain size increases as the film thickness and temperature increase.

The decrease of activation energy with increase in film thickness can also be attributed to the change in the barrier height due to the change in grain size of the polycrystalline film. It has been established (Chopra 1969) that the grain size is approximately proportional to thickness and hence, increases as the thickness increases, which results in the reduction of the barrier width. It has also been observed from XRD and SEM results that grain size increases as thickness increases. The reduction in barrier width will reduce the energy needed to activate the charge carriers for conduction.

The curves are seen to exhibit two distinct regions, one below $383 \mathrm{~K}$ and the other above $383 \mathrm{~K}$. The calculated activation energy is found to be in the range of $0 \cdot 09-0 \cdot 0105 \mathrm{eV}$ at low temperature region (below $383 \mathrm{~K}$ ) and $0.267-0.144 \mathrm{eV}$ at high temperature region (above $383 \mathrm{~K}$ ). The estimated values of activation energy increase at high temperature region (transition temperature) for all the films. This may be attributed to transition of conduction through the film from electron to hole conduction. The transition of conduction from $n$-type to $p$-type may be due to the increase of migration of tellurium vacancies with temperature. As-deposited film is ' $n$ ' type which is confirmed by hot-probe method. The broken bonds at the grain boundaries generate acceptor states in $n$-type films, capture electrons from the interior of the grains and give rise to $p$-type inversion layers between adjacent grains. In the nanostructure $n$-type films with high density of grain boundaries, thermally-activated hole conductivity becomes dominant and leads to its inversion from $n$ to $p$ type. Similar observations have been reported by Abd El-Ati (1997) for PbTe thin films and Dashevsky et al (2005) for the electrical conductivity of indium-doped PbTe thin films. The transition of conductivity observed by the effective free path model vindicates the above experimental results.

\subsection{Optical properties}

The optical absorption spectrum of the nanocrystalline PbTe thin films of different thicknesses is shown in figure 5. Excitonic absorption peak for nanocrystalline PbTe thin films of different thicknesses are detected at about 498.4, 676.2 and $1021.2 \mathrm{~nm}$, whereas PbTe bulk excitonic absorption band appears at $\sim 3859 \mathrm{~nm}$. Such a large blue shift of excitonic absorption band can be attributed to the small crystallite size of the samples and hence, the result of quantization effect.

The bandgap energy ' $E_{\mathrm{g}}$ ' of the samples are calculated using the relation:

$$
\alpha h v=A\left(h v-E_{\mathrm{g}}\right)^{n},
$$

where $h v$ is the photon energy, $A$ and $n$ are constants. For allowed direct transitions, $n=1 / 2$. From $(\alpha h v)^{2}$ vs $h v$ plots presented in figures 6-8, direct bandgap of the samples of different thicknesses were determined. The bandgap is estimated by extrapolating the linear portion to the energy axis

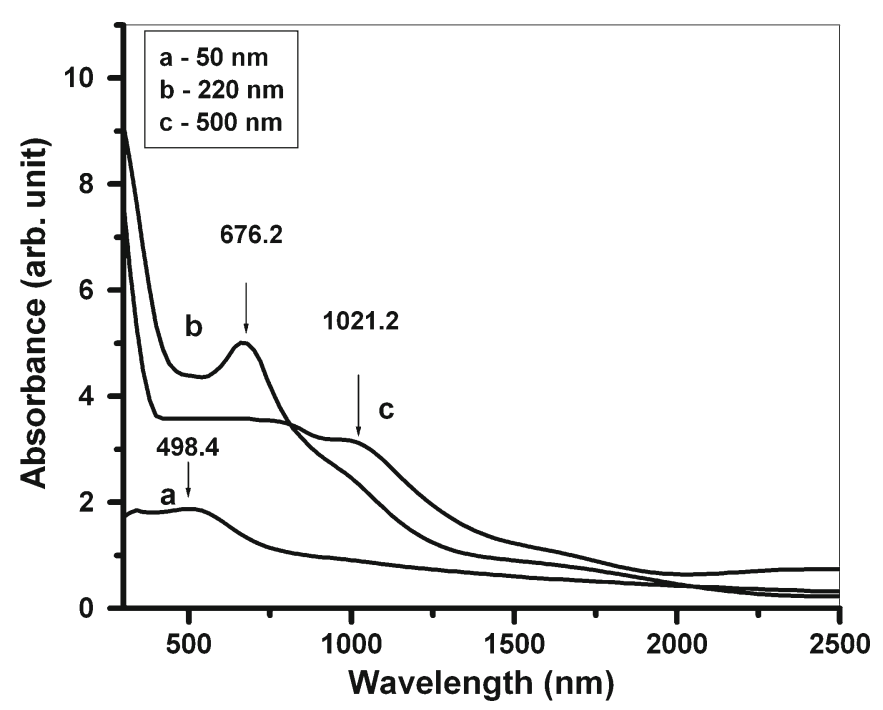

Figure 5. Optical absorption spectrum of nanocrystalline PbTe thin films. 


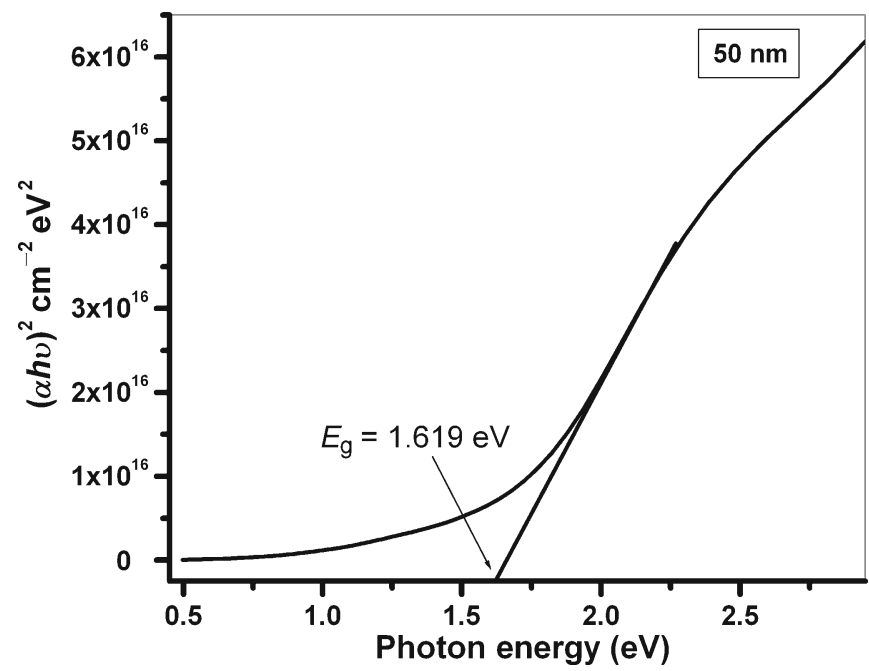

Figure 6. $(\alpha h v)^{2}$ vs $h v$ plot for PbTe nanocrystalline thin film of thickness $50 \mathrm{~nm}$.

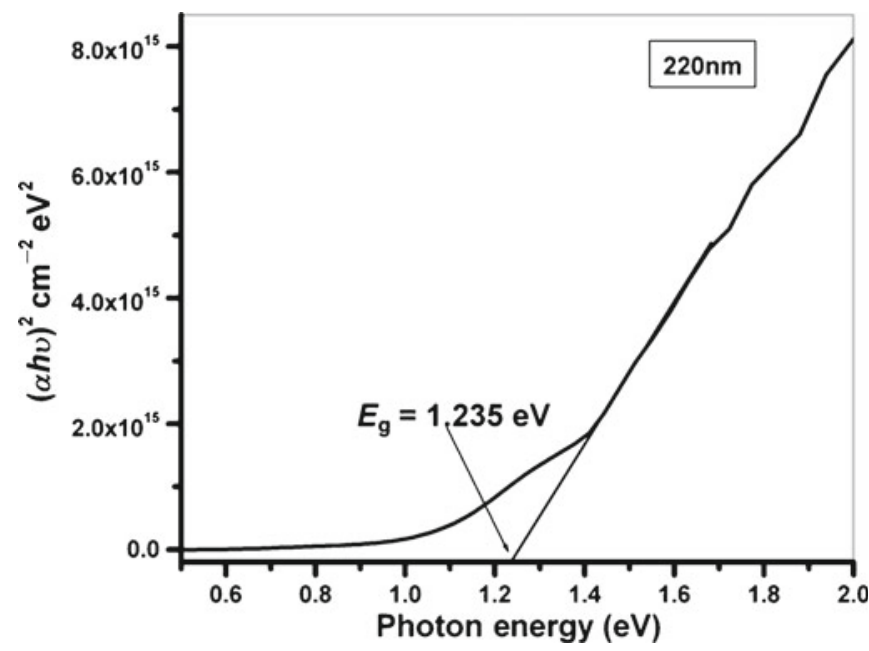

Figure 7. $(\alpha h v)^{2}$ vs $h v$ plot for PbTe nanocrystalline thin film of thickness $220 \mathrm{~nm}$.

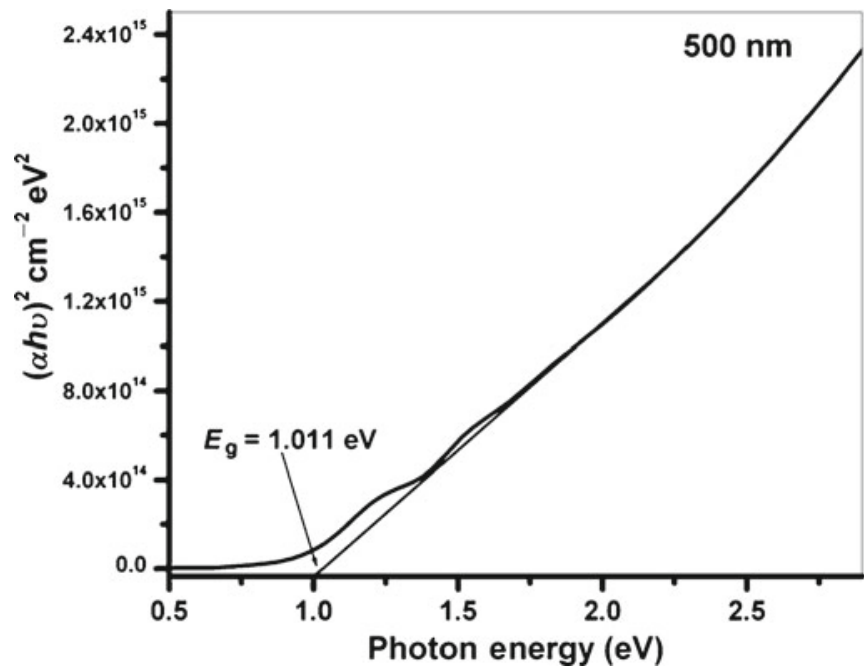

Figure 8. $(\alpha h v)^{2}$ vs $h v$ plot for PbTe nanocrystalline thin film of thickness $500 \mathrm{~nm}$. and is found to be $1.61,1.23$ and $1.01 \mathrm{eV}$ for the nanocrystalline $\mathrm{PbTe}$ thin films of different morphologies such as nanoparticles, worm-like and nanocubes, respectively. It is worth pointing out that the observed optical bandgap energy in nanocrystalline $\mathrm{PbTe}$ films is much larger than the bulk value of $0.32 \mathrm{eV}$ due to quantum confinement effect. From XRD results, grain size of the film is in the range of 27 $43 \mathrm{~nm}$, which is much smaller than the exciton Bohr radius of $150 \mathrm{~nm}$ in $\mathrm{PbTe}$. Therefore, PbTe nanocrystallites in the films can lead to a strong quantum confinement effect, which may account for the optical bandgap energy increase. The quantum confinement effect in polycrystalline $\mathrm{PbTe}$ thin films has also been observed by Lawson et al (1960), who reported increasing blue shift of photoconductivity onset as the average grain size of PbTe thin films decrease. Cao et al (2006) also observed a large blue shift of the absorption edge in UV region $(5.04-4.57 \mathrm{eV})$ for their synthesized $\mathrm{PbS}$ nanocubes due to its small effective mass. Li et al (2004) reported large blue shift at about $1.97 \mathrm{eV}$ for PbSe nanorods due to size quantization effects. Zhao and Qi (2006) observed a relative blue shift in the absorption spectrum of star-shaped $\mathrm{PbS}$ nanocrystals of $\sim 100 \mathrm{~nm}$ size and attributed that it is caused from quantum-size effects in the pointed tips of the star structures. From the above research reports, we believe that in addition to the small crystallite size, different morphologies of the prepared samples also play a vital role for the increase of optical bandgap energy. It is important that we are reporting a large blue shift in nanocrystalline PbTe thin films of different morphologies for the first time. This structure induced change in optical properties may have potential applications in optoelectronic devices.

\subsection{Photoconduction}

The variation of photocurrent with applied voltage as a function of light intensity is presented in figure 9. The observed

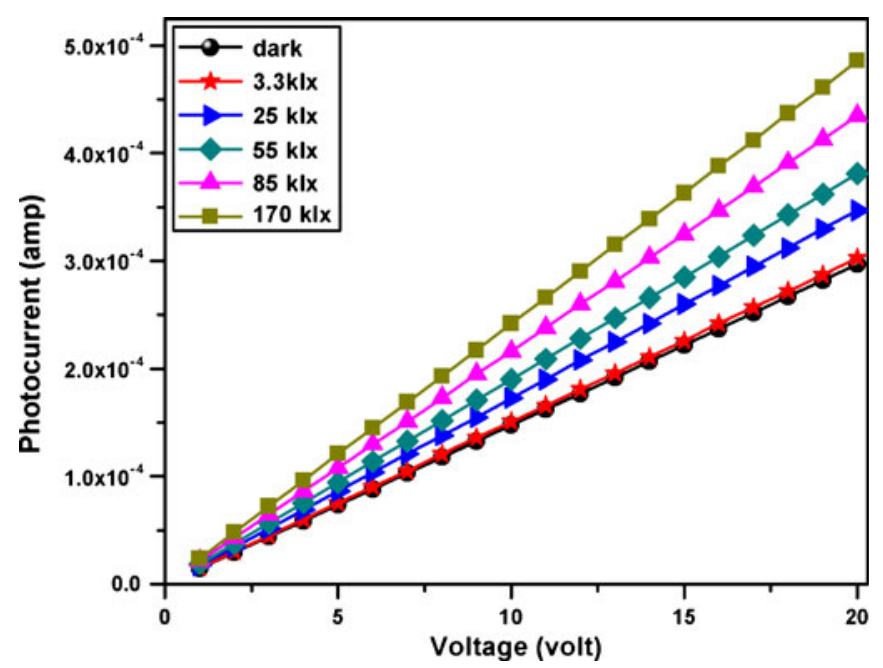

Figure 9. Dependence of photocurrent with applied voltage for different light intensities. 


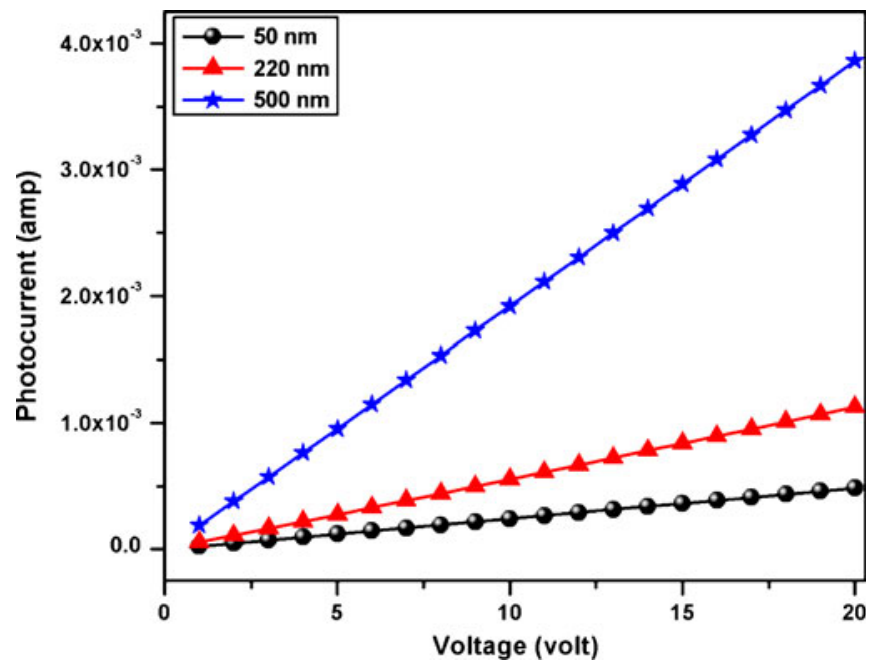

Figure 10. Variation of photocurrent with applied voltage for different film thicknesses.

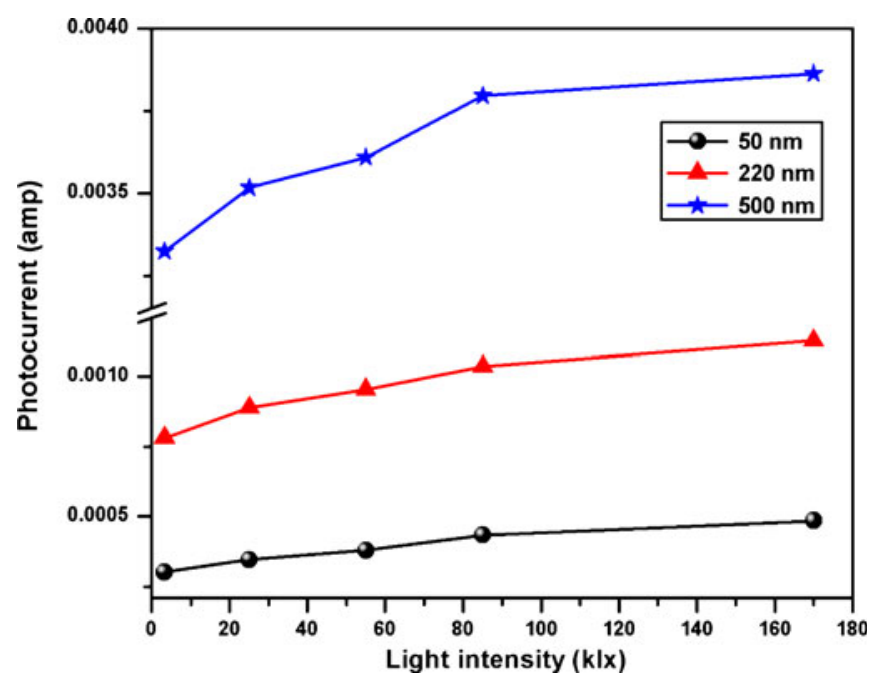

Figure 11. Photocurrent as a function of light intensity for different thicknesses.

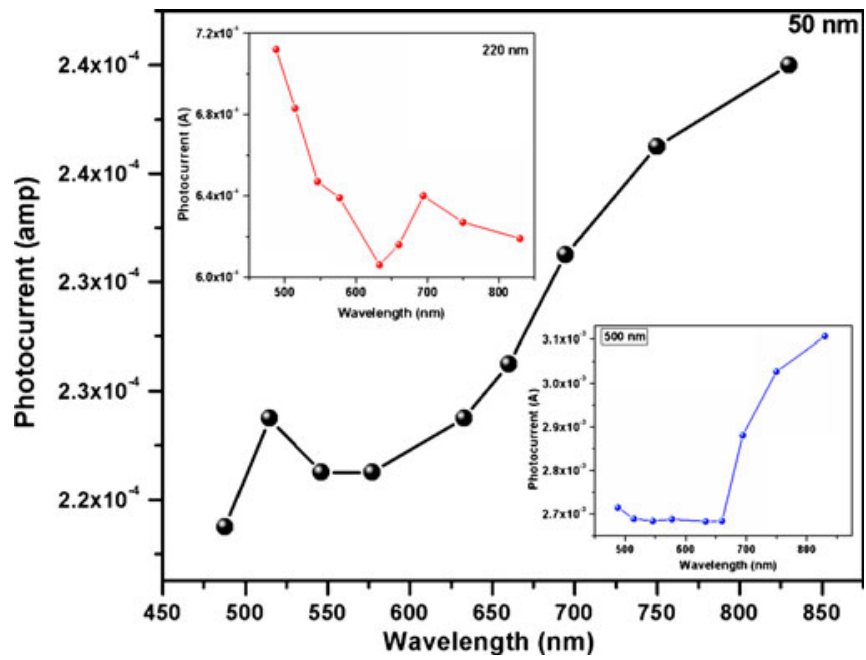

Figure 12. Spectral dependence of photocurrent for different thicknesses. photocurrent increases with increase in applied voltage as well as light intensity. The increase in photocurrent with light intensity may be mainly due to lowering of intercrystalline potential barriers rather than increase in the free carrier concentration (Green and Miles 1973). The photocurrent increases linearly with increase in applied voltage which indicates ohmic conduction. Figure 10 presents variation of photocurrent with applied voltage for different film thicknesses for $170 \mathrm{klx}$ light intensity. The photocurrent increases with increase in film thickness and applied voltage. The value of photocurrent at $20 \mathrm{~V}$ for the film thickness of $500 \mathrm{~nm}$ is $3.86 \times 10^{-3} \mathrm{~A}$, which is one order higher than the film thickness, $50 \mathrm{~nm}\left(4.86 \times 10^{-4} \mathrm{~A}\right)$. Lin et al (2012) observed photocurrent $1.2 \times 10^{-5} \mathrm{~A}$ for PbTe nanocrystal thin film. As compared with the reported value, the observed value is one to two orders higher which may be attributed to the existence of different morphologies of the films and also due to the improvement in crystallinity. Figure 11 depicts dependence of photocurrent with light intensity for different film thicknesses. The photocurrent rises with increase in light intensity and also with film thickness. It clearly indicates the influence of film thickness on photoconductivity. The spectral dependence of photocurrent for different film thicknesses is presented in figure 12. It is observed that the value of photocurrent at $830 \mathrm{~nm}$ is $2.4 \times 10^{-4} \mathrm{~A}, 6.14 \times 10^{-4} \mathrm{~A}$ and $3.1 \times 10^{-3} \mathrm{~A}$ for the films thickness of 50,220 and $500 \mathrm{~nm}$, respectively. Interestingly, maxima peak shifts to higher wavelength with increase in film thickness. For the film thickness of $50 \mathrm{~nm}$, the maximum photocurrent observed at $514.5 \mathrm{~nm}$ is closer to the observed value of the absorption spectrum. Similar observation is noticed for higher film thicknesses also.

\section{Conclusions}

The films studied are nanocrystalline having rock salt structure. As thickness increases, there is an increase in grain size and decrease in strain as well as dislocation density. These results indicate that the degree of crystallinity of the films increases with film thickness. SEM analysis indicates that the different morphology of PbTe films such as nanoparticles, worm-like and nanocubes were obtained by tuning the thickness of the films. In all films, an inversion of the type of conductivity takes place leading to a thermally activated conductivity. The prepared nanocrystalline PbTe thin films exhibit good photoconductivity and this may be due to the existence of different morphologies of the films and also due to the improvement in crystallinity. Our results show that nanocrystalline $\mathrm{PbTe}$ thin films of different morphologies with large blue shift can be prepared and are promising materials for potential application in optical devices. To our knowledge, this is the first time we demonstrate the preparation of nanocrystalline $\mathrm{PbTe}$ thin films with different morphologies by using a simple thermal evaporation process. 


\section{Acknowledgment}

One of the authors ( $\mathrm{L} \mathrm{K}$ ) would like to acknowledge the Council of Scientific and Industrial Research (CSIR), India, for awarding a Senior Research Fellowship (SRF) to carry out this work.

\section{References}

Abd El-Ati M I 1997 Phys. Solid State 3968

Brown R W, Miller A R and Allgaier R S 1970 Thin Solid Films 5157

Cao H, Wang G, Zhang S and Zhang X 2006 Nanotechnology 17 3280

Chopra K L 1969 Thin film phenomena (New York: McGraw Hill Publications)

Damodara Das V and Bahulayan C 1996 Thin Solid Films 27455

Dashevsky Z, Kreizman R and Dariel M P 2005 J. Appl. Phys. 98 094309

Green Mino and Miles R E 1973 J. Phys. D: Appl. Phys. 6 L45

Hema Chandra C, Naveen Kumar J, Madhusudhana Rao N and Uthanna S 2007 J. Cryst. Growth 30668
Kumar S, Khan Z H and Majeed Khan M A 2005 Curr. Appl. Phys. 5561

Kungumadevi L, Rajasekar K, Subbarayan A and Sathyamoorthy R 2008 Ionics 1463

Lawson W D, Smith F A and Young A S 1960 J. Electrochem. Soc. 107206

Lee H W, Lau S P, Wang Y G, Tse K Y and Hang H H 2004 J. Cryst. Growth 268598

Li L, Wu Q and Ding Y 2004 Nanotechnology 151877

Lin Zhonghai, Wang Minqiang, Zhang Liyuan and Xue Yaohui 2012 J. Mater. Chem. 229082

Ma W, Luther J M, Zheng H, Wu Y and Alivisatos A P 2009 Nano Lett. 91699

Mandale A B 1991 Thin Solid Films 19515

Mayadas A F and Shatzkes M 1970 Phys. Rev. B1 1382

Seetharama Bhat K and Damodara Das V 1985 Phys. Rev. B32 6713

Seto J Y W 1975 J. Appl. Phys. 465247

Sondheimer E H 1950 Phys. Rev. 80401

Tellier C R 1978 Thin Solid Films 51311

Wang J, Hu J, Sun X and Agarwal A M 2008 J. Appl. Phys. 104 053707

Zhang W, Liu Z, Zheng C, Xu C, Liu Y and Wang G 2003 Mater. Lett. 572755

Zhang Q, Huang F and Li Y 2005 Coll. Surf. A257-258 497

Zhao N and Qi L 2006 Adv. Mater. 18359 